\title{
Weak Gravitational Lensing by Horndeski Theory Using the Gauss-Bonnet Theorem
}

\author{
Wajiha Javed, ${ }^{1, *}$ Jameela Abbas, ${ }^{2, \dagger}$ Yashmitha Kumaran, ${ }^{3, \ddagger}$ and Ali Övgün ${ }^{4,3, \S}$ \\ ${ }^{1}$ Division of Science and Technology, University of Education, Township-Lahore, Pakistan \\ ${ }^{2}$ Department of Mathematics, University of Education, \\ Township, Lahore-54590, Pakistan. \\ ${ }^{3}$ Physics Department, Arts and Sciences Faculty, Eastern Mediterranean University, Famagusta, North Cyprus via Mersin 10, Turkey. \\ ${ }^{4}$ Instituto de Física, Pontificia Universidad Católica de Valparaíso, Casilla 4950, Valparaíso, Chile.
}

(Dated: January 7, 2020)

\begin{abstract}
The main goal of this paper is to study the weak gravitational lensing by Horndeski black hole in weak field a pproximation. In order to do so, we exploit the Gibbons-Werner method to the optical geometry of Horndeski black hole and implement the Gauss-Bonnet theorem to accomplish the deflection angle of light in weak field re gion. Furthermore, we have endeavored to extend the scale of our work by comprising the impact of plasma medium on the deflection angle as properly. Later, the graphical influence of the deflection angle of photon on Horndeski black hole in plasma and nonplasma medium is examined.
\end{abstract}

PACS numbers: 95.30.Sf, 98.62.Sb, 97.60.Lf

Keywords: weak gravitational lensing; Horndeski black hole; deflection angle; Gauss-Bonnet theorem

\section{INTRODUCTION}

Gravitational physics has been an area of interest for centuries among physicists from diverse disciplines. Substantiating Lorentz Transformations and General Theory of Relativity encouraged many researchers to reform Newtonian gravity in an attempt to surpass it's limitations on high speeds, extreme gravity and particle nature. The most triumphant vindication to the Newtonian "missing mass" problem is Dark Matter hypothesis [1]. While this theory has numerous merits, there were disputes on introducing a new form of matter, suggesting the essential modification of Newtonian dynamics to account for the same. Hitherto, the idea of Modified Gravity was proposed, emerging as the widely accredited competitor of General Relativity. But, due to theoretical complexities and observational inadequacies, this approach is transcended by the Scalar-tensor Theory; it efficiently consolidates the scalar field (mass) with the metric (gravity) in the recent years [2-9].

Conversely, cluster of mass bending light to create a lensing effect - thus, acting as a magnifying glass and revealing inconspicuous details - validated the existence of dark matter [10-13]. This phenomena, known as gravitational lensing, is categorised into strong lensing, weak lensing and micro-lensing [14-52].

In this paper, we are interested in weak lensing in which the distortions of the light source are high enough to be just detected, but too low to be able to promptly determine it's salient features. The typical mode of perpetuation is to calculate the angle of deflection of light, and herein, Gauss-Bonnet theorem is employed, mathematically expressed as: [53]

$$
\iint_{D} K d S+\int_{\partial D} \kappa d t+\sum_{i} \alpha_{i}=2 \pi \chi(D)
$$

where, $K$ is the Gaussian curvature, $\kappa$ is the geodesic curvature, $\alpha_{i}$ is the exterior angle with $i^{\text {th }}$ vertex, and $\chi$ is the Euler characteristic of topology, altogether defined with $g$ as the Riemannian metric of the manifold of lens in a surface domain $(D, \chi, g)$ outside the light trajectory. Some other works can be found in Refs. [54-93]. For an asymptotically flat line-of-sight, the deflection angle, is given by: [53]

$$
\hat{\alpha}=-\iint_{D} K d S
$$

\footnotetext{
*Electronic address: wajiha.javed@ue.edu.pk; wajihajaved84@yahoo.com

$\dagger$ Electronic address: jameelaabbas30@gmail.com

‡Electronic address: y.kumaran13@gmail.com

$\S$ Electronic address: ali.ovgun@pucv.cl
} 
With this method as the basis, consider a black hole that obeys the most generalised Scalar-tensor theory in fourdimensional spacetime: the Horndeski theory - it characterizes the scalar field as a new degree of freedom, evolving into a Lagrangian that unravels second-order field equations of motion. The action is written as: [94, 95]

$$
S=\int d^{4} x \sqrt{-g} \mathcal{L}
$$

with the generalised Galilean Lagrangean, $\mathcal{L} \equiv\left(\mathcal{L}_{2}+\mathcal{L}_{3}+\mathcal{L}_{4}+\mathcal{L}_{5}\right)$ expanded such that:

$$
\begin{aligned}
& \mathcal{L}_{2}=G_{2} \\
& \mathcal{L}_{3}=-G_{3} \square \phi \\
& \mathcal{L}_{4}=G_{4} R+G_{4 X}\left[(\square \phi)^{2}-\left(\nabla_{\mu} \nabla_{\nu} \phi\right)^{2}\right] \\
& \mathcal{L}_{5}=G_{5} G_{\mu \nu} \nabla^{\mu} \nabla^{\nu} \phi-\frac{G_{5 X}}{6}\left[(\square \phi)^{3}-3 \square \phi\left(\nabla_{\mu} \nabla_{\nu} \phi\right)^{2}+2\left(\nabla_{\mu} \nabla_{\nu} \phi\right)^{3}\right]
\end{aligned}
$$

It is to be noted that $G_{i}$ is an arbitrary function of scalar field, $\phi$ and kinetic energy, $X \equiv-\partial_{\mu} \phi \partial^{\mu} \phi / 2$, whereas, the subscript $X$ denotes the derivative with respect to $X$. Also, $g_{\mu \nu}$ is the metric tensor with a determinant $g$, $R$ is the Ricci scalar, $G_{\mu \nu}$ is the Einstein tensor, along with:

$$
\square \phi \equiv g^{\mu \nu} \nabla_{\mu} \nabla_{\nu} \phi \quad ; \quad\left(\nabla_{\mu} \nabla_{\nu} \phi\right)^{2} \equiv \nabla_{\mu} \nabla_{\nu} \phi \nabla^{\mu} \nabla^{\nu} \phi \quad ; \quad\left(\nabla_{\mu} \nabla_{\nu} \phi\right)^{3} \equiv \nabla_{\mu} \nabla_{\nu} \phi \nabla^{\nu} \nabla^{\sigma} \phi \nabla_{\sigma} \nabla_{\mu} \phi
$$

Building on this premise, this paper is organized as follows: In section 2, we review some basic concepts about Horndeski BH. In section 3, we evaluate the Gaussian optical curvature for deflection angle and compute the deflection angle by applying GBT. In section 4, the deflection angle of light for Horndeski BH in a plasma medium is obtained. The last section comprises of concluding remarks.

\section{EVALUATION OF PHOTON LENSING FOR HORNDESKI BLACK HOLES}

We consider the particular case of the action in which $G_{2}=\eta X, G_{4}=\zeta+\beta \sqrt{-X}$ and $G_{3}=G_{5}=0$, where $\eta$ and $\beta$ are dimensionless parameters and $\zeta=M_{p l}^{2} /(16 \pi)$. Then, the action takes the explicit form

$$
S=\int \sqrt{-g} d^{4} x\left\{\left[\zeta+\beta \sqrt{\frac{\partial \phi)^{2}}{2}}\right] R-\frac{\eta}{2}(\partial \phi)^{2}-\frac{\beta}{\sqrt{2(\partial \phi)^{2}}}\left[(\phi)^{2}-\left(\nabla_{\mu} \nabla_{\nu} \phi\right)^{2}\right]\right\} .
$$

The coefficient $\zeta$ gives the Einstein-Hilbert part of the action; one of the parameters $\eta$ and $\beta$ can be absorbed into the scalar field by means of a redefinition, but we will not do it in order to trace the origin of the different terms. The field equations resulting from Eq.(9) admit a static, spherically symmetric, and asymptotically flat solution of the form [95]

$$
d s^{2}=-A(r) d t^{2}++B(r) d r^{2}+C(r)\left(d \theta^{2}+\sin ^{2} \theta d \phi^{2}\right)
$$

With

$$
A(r)=1-\frac{\mu}{r}-\frac{\beta^{2}}{2 \zeta \eta r^{2}}, B(r)=\frac{1}{A(r)}, C(r)=r^{2}
$$

The integration constant $\mu$ can be interpreted as twice the black hole mass, i.e $\mu=2 M$. The parameters $\beta$ and $\eta$ should share the same sign and scalar field is given by

$$
\begin{aligned}
\phi(r)= & \pm 2 \sqrt{\frac{\zeta}{\eta}}\left\{\arctan \left[\frac{\beta^{2}+\zeta \eta \mu r}{\beta \sqrt{2 \zeta \eta r(r-\mu)-\beta^{2}}}\right]-\arctan \left(\frac{\mu}{\beta} \sqrt{\frac{\zeta \eta}{2}}\right)\right\}, \\
& \text { if } \beta>0 \text { and } \eta>0 \\
\phi(r)= & \pm 2 \sqrt{\frac{\zeta}{-\eta}}\left\{\arctan \left[\frac{\beta^{2}+\zeta \eta \mu r}{\beta^{2}-\beta \sqrt{2 \zeta \eta r(r-\mu)}}\right]+\arctan \left(\frac{\mu}{\beta} \sqrt{\frac{-\zeta \eta}{2}}\right)\right\}, \\
& \text { if } \beta<0 \text { and } \eta<0
\end{aligned}
$$


The geometry is singular as the origin of coordinates. It is useful to define the parameter $\gamma=\beta^{2} /(2 \zeta \eta)$ in order to simplify the notation. We can see that a negative value of $\gamma$ makes this metric identical to the Reissner-Nordstrom metric, with the squared charge given by $Q^{2}=-\gamma$. Adimensionalizing with $\mu$, we let $x=r / \mu, \tilde{t}=t / \mu$ and $\tilde{\gamma}=\gamma / \mu^{2}$, so that the metric functions become

$$
A(x)=1-\frac{1}{x}-\frac{\tilde{\gamma}}{x^{2}}, \quad B(x)=\frac{1}{x}, \quad C(x)=x^{2} .
$$

The radius of the event horizon is obtained as the largest solution of the equation $A(x)=0$, to give $x_{h}=(1+$ $\sqrt{1+4 \tilde{\gamma}}) / 2$. Therefore, to avoid a naked singularity we must have $\tilde{\gamma} \geq-1 / 4$, with $\tilde{\gamma}=-1 / 4$ in the case of an extremal black hole. Correspondingly, we work with within the interval $[-1 / 4,+\infty)$.

Now to acquire the null geodesics $\left(d s^{2}=0\right)$, the $\mathrm{BH}$ optical spacetime can be simply written in equatorial plane $\theta=\frac{\pi}{2}$ :

$$
d t^{2}=\frac{B(x)}{A(x)} d x^{2}+\frac{C(x)}{A(x)} d \phi^{2} .
$$

The non-zero Christoffel symbols related to the above optical metric are:

$$
\begin{aligned}
\Gamma_{x x}^{x} & =-\frac{A^{\prime}(x)}{A(x)}, \Gamma_{\phi \phi}^{x}=\frac{A^{\prime}(x) C(x)}{2}-\frac{A(x) C(x)^{\prime}}{2}, \\
\Gamma_{x \phi}^{\phi} & =\frac{C(x)}{2 C(x)}-\frac{A^{\prime}(x)}{2 A(x)} .
\end{aligned}
$$

The Gaussian curvature that is proportional to the Ricci scalar can be defined as:

$$
\mathcal{K}=\frac{R_{\text {icciScalar }}}{2}
$$

using the non-zero christopher symbol the Gaussian optical curvature for Horndeski BH can be computed as:

$$
\mathcal{K}=\frac{A^{2}(x) C^{\prime}(x)^{2}}{4 C(x)^{2}}-\frac{A^{\prime}(x)^{2}}{4}-\frac{A(x)^{2} C^{\prime \prime}(x)}{2 C(x)}+\frac{A(x) A^{\prime \prime}(x)}{2},
$$

after simplification, we obtain

$$
\mathcal{K}=-\frac{1}{x^{3}}+\frac{3}{4 x^{4}}(1-4 \tilde{\gamma})+\frac{3 \tilde{\gamma}}{x^{5}}+\frac{2 \tilde{\gamma}^{2}}{x^{6}}+\mathcal{O}\left(x^{7}\right)
$$

Let us bear in mind the GBT for a two dimensional manifold. Regarding to this, we take into account a regular domain $M_{R}$ arranged by 2-dimensional surface $S$ with Riemannian metric $\hat{g}_{i j}$, in conjunction with boundary $\partial M_{R}=$ $\gamma_{g} \cup C_{R}$, so GBT allows a coupling betwixt the geometry and topology in terms of the following relation

$$
\iint_{M_{R}} \mathcal{K} d S+\oint_{\partial M_{R}} k d \sigma+\sum_{j} \hat{\theta}_{j}=2 \pi \mathcal{X}\left(M_{R}\right)
$$

Here $\mathcal{K}$ represented as the Gaussian optical curvature. It is a well-known fact that for regular domain the Euler characteristic $\mathcal{X}_{M_{R}}=1$, while $k$ is known as a geodesic curvature and is defined as [96]

$$
k=g^{o p}\left(\nabla_{\dot{\gamma}} \dot{\gamma}, \ddot{\gamma}\right)
$$

having the unit speed condition $g^{o p}(\dot{\gamma}, \dot{\gamma})=1$, where $\ddot{\gamma}$ is the unit acceleration vector. In the case of $R \rightarrow \infty$, the corresponding jump angles are taken as $\pi / 2$ (in short $\theta_{O}+\theta_{S} \rightarrow \pi$ ). using the fact that, from geodesic there is a zero contribution i.e. $k\left(\gamma_{\tilde{g}}\right)=0$, we shall pursue a contribution by virtue of the curve $C_{R}$ and this contribution can be computed as

$$
k\left(C_{R}\right)=\left|\nabla_{\dot{C}_{R}} \dot{C}_{R}\right|
$$

Let us consider $C_{R}:=r(\phi)=R=$ const, while $R$ endows the distance from the coordinate origin. The radial component of the geodesic curvature states as

$$
\left(\nabla_{\dot{C}_{R}} \dot{C}_{R}\right)^{r}=\dot{C}_{R}^{\phi}\left(\partial_{\phi} \dot{C}_{R}^{\phi}\right)+\Gamma_{\phi \phi}^{r}\left(\dot{C}_{R}^{\phi}\right)^{2}
$$


Doing the analogous identifications among the frequencies, energy and mass, which implies the form invariant quantity

$$
\lim _{R \rightarrow \infty}\left[k \frac{d \sigma}{d \phi}\right]_{C_{R}}=1
$$

ensuing in this manner the form invariant deflection angle

$$
\Theta=-\int_{0}^{\pi} \int_{b / \sin \phi}^{\infty} \mathcal{K} d S .
$$

This equation inscribe the global impact on the lensing of particles on account of the fact that one has to integrate over the optical domain of integration outside the enclosed mass. Where

$$
\begin{aligned}
d S & =\sqrt{\operatorname{det} g} d r d \phi \\
& \simeq\left(\frac{3}{2}+x+\frac{3 \tilde{\gamma}}{x}\right) d r d \phi .
\end{aligned}
$$

Now, by using equation 2.10 into 2.16 we find the deflection angle for Horndeski BH.

$$
\Theta=\frac{2 \mu^{2}}{b}+\frac{3 \pi \mu \tilde{\gamma}}{4 b^{2}}+\frac{3 \pi \mu^{3}}{16 b^{2}}+\frac{4 \mu^{2} \tilde{\gamma}}{3 b^{3}}+\mathcal{O}\left(\mu^{4}, b^{4}\right) .
$$

\section{GRAPHICAL ANALYSIS}

This section is devoted to scrutinize the graphical effect of deflection angle $\Theta$ on Horndeski BHs. Furthermore, describe the physical eminence of these plots by examining the stable and unstable state of BH to analyze the impact of $\tilde{\gamma}, \mu$ and impact parameter $b$ on deflection angle.

\section{A. Deflection angle over Impact parameter}

This subsection provides the analysis of deflection angle $\Theta$ with impact parameter $b$ for different values of $\tilde{\gamma}$ and $\mu$.
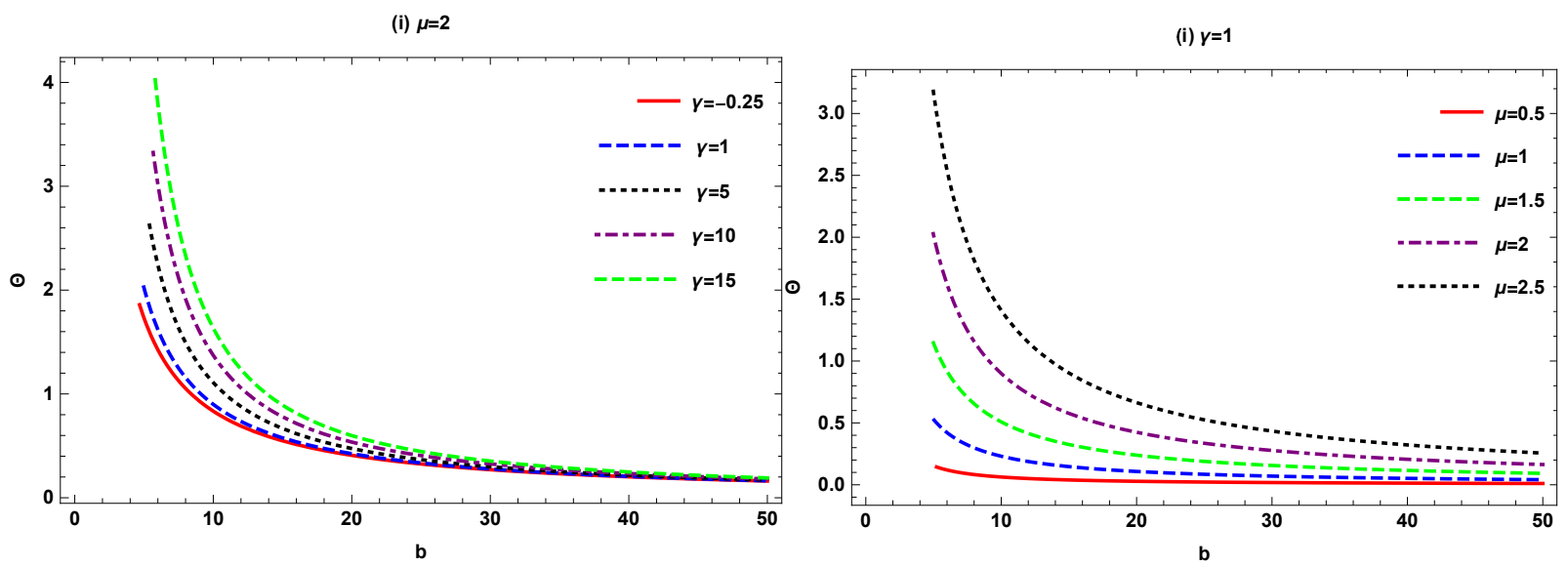

Figure 1: $\Theta$ versus $b$.

- Figure 1 exhibits the effect of $\Theta$ versus $b$ for different parameters. It is to be analyzed that deflection angle has an indirect relation with impact parameter and one can choose the $b \in[1,50]$ for stable behavior.

1. Figure (i), display the graphical influence of deflection angle with $b$ by varying $\tilde{\gamma}$ and by fixing $\mu$. For this, we observe that negative $\tilde{\gamma}$ makes this metric identical to RN solution. It is to be investigated that if $\tilde{\gamma}$ increase then deflection angle exponentially increase. We analyze the stable behavior only for $\tilde{\gamma} \in$ $[-1 / 4, \infty]$.

2. Figure (ii), depicts that the deflection angle gradually increase by increasing the mass term. 


\section{B. Weak deflection angle in a plasma medium}

With the objective to mainstream the impact of plasma, keep in mind the case whilst light travels from vacuum to a hot, ionized gas medium. Consider $v$ be the velocity of photon within plasma. The refractive index, $n(x)$ is defined by: [73]

$$
n(x) \equiv \frac{c}{v}=\frac{1}{d x / d t} \quad(c=1)
$$

The equation for refractive index by Horndeski BH is computed as [73],

$$
n(x)=\sqrt{1-\frac{\omega_{e}^{2}}{\omega_{\infty}^{2}} A(x)}
$$

here $\omega_{e}$ referred to as electron plasma frequency and $\omega_{\infty}$ introduced as photon frequency found by an observer at infinity. Therefore, the equivalent optical metric designated as:

$$
d \sigma^{2}=g_{j k}^{o p t} d x^{j} d x^{k}=\frac{n^{2}(x)}{A(x)}\left[\frac{1}{A(x)} d x^{2}+C(x) d \phi^{2}\right] .
$$

where the metric functions of the above optical metric are defined in Eq. 2.6

The non-zero Christoffel symbol corresponding to Eq. 2.21 are defined as

$$
\begin{aligned}
& \Gamma_{x x}^{x}=\left(1+\frac{\omega_{e}^{2}}{\omega_{\infty}^{2}} A(x)\right)\left[-A^{\prime}(x) A^{-1}(x)\left(1-\frac{\omega_{e}^{2}}{\omega_{\infty}^{2}} A(x)\right)-\frac{A^{\prime}(x)}{2} \frac{\omega_{e}^{2}}{\omega_{\infty}^{2}}\right] \\
& \Gamma_{\phi \phi}^{x}=\left(1+\frac{\omega_{e}^{2}}{\omega_{\infty}^{2}} A(x)\right)\left[\left(1-\frac{\omega_{e}^{2}}{\omega_{\infty}^{2}} A(x)\right)\left\{\frac{A^{\prime}(x) C(x)}{2}-\frac{C^{\prime}(x) A(x)}{2}\right\}+\frac{A^{\prime}(x) A(x) C(x)}{2} \frac{\omega_{e}^{2}}{\omega_{\infty}^{2}}\right] \\
& \Gamma_{x \phi}^{\phi}=\left(1+\frac{\omega_{e}^{2}}{\omega_{\infty}^{2}} A(x)\right)\left[\left(1-\frac{\omega_{e}^{2}}{\omega_{\infty}^{2}} A(x)\right)\left\{\frac{C^{\prime}(x) C^{-1}(x)}{2}-\frac{A^{\prime}(x) A^{-1}(x)}{2}\right\}-\frac{A^{\prime}(x)}{2} \frac{\omega_{e}^{2}}{\omega_{\infty}^{2}}\right]
\end{aligned}
$$

The optical Gaussian curvature for the above optical metric is calculated by using Eq. 2.6 as follows

$$
\begin{aligned}
\mathcal{K}= & -\frac{3}{4 x^{3}}+\frac{1}{2 x^{4}}(1-\tilde{\gamma})+\frac{\tilde{\gamma}}{4 x^{5}}+\frac{3 \tilde{\gamma}^{2}}{2 x^{6}}-\frac{3}{4 x^{3}}\left(\frac{\omega_{e}^{2}}{\omega_{\infty}}\right)-\frac{1}{4 x^{3}}\left(\frac{\omega_{e}^{2}}{\omega_{\infty}}\right) \\
+ & \frac{1}{2 x^{4}}\left(\frac{5}{4}+\tilde{\gamma}\right)\left(\frac{\omega_{e}^{2}}{\omega_{\infty}}\right)+\frac{1}{x^{5}}\left(\frac{1}{8}+3 \tilde{\gamma}\right)\left(\frac{\omega_{e}^{2}}{\omega_{\infty}}\right)+\frac{\tilde{\gamma}}{x^{6}}\left(-\frac{5}{8}+\frac{11 \tilde{\gamma}}{2}\right) \\
& \left(\frac{\omega_{e}^{2}}{\omega_{\infty}}\right)-\frac{\tilde{\gamma}^{2}}{x^{7}}\left(\frac{\omega_{e}^{2}}{\omega_{\infty}}\right)
\end{aligned}
$$

Hence, we get differently which goes to $\alpha$ :

$$
\left.\lim _{x \rightarrow \infty} k_{g} \frac{d \tilde{\sigma}}{d \varphi}\right|_{C_{R}}=\alpha .
$$

We use straight line approximation $r=b / \sin \varphi$, for the limit $x \rightarrow \infty$, then GBT stated as [73]

$$
\left.\lim _{x \rightarrow \infty} \int_{0}^{\pi+\Theta}\left[k_{g} \frac{d \tilde{\sigma}}{d \varphi}\right]\right|_{C_{R}} d \varphi=\pi-\lim _{x \rightarrow \infty} \int_{0}^{\pi} \int_{b / \sin \varphi}^{x} \mathcal{K} d S
$$

After simplification, we obtain

$$
\begin{aligned}
\Theta & \simeq \frac{2 \mu^{2}}{b}+\frac{\mu \pi \tilde{\gamma}}{8 b^{2}}-\frac{\mu^{2} \tilde{\gamma}}{18 b^{3}}+\frac{3 \tilde{\gamma}^{2}}{2 b^{3}}-\frac{\mu^{3} \pi}{8 b^{2}}-\frac{\mu^{2} \omega_{e}^{2}}{\omega_{\infty}^{2}}-\frac{\mu^{3} \pi}{2 b^{2}} \frac{\omega_{e}^{2}}{\omega_{\infty}^{2}} \\
& -\frac{\mu \pi \tilde{\gamma}}{4 b^{2}} \frac{\omega_{e}^{2}}{\omega_{\infty}^{2}}-\frac{15 \mu^{2} \tilde{\gamma}}{9 b^{3}} \frac{\omega_{e}^{2}}{\omega_{\infty}^{2}}+\frac{\tilde{\gamma}^{2}}{18 b^{3}} \frac{\omega_{e}^{2}}{\omega_{\infty}^{2}}+\mathcal{O}\left(\mu^{4}, b^{4}\right) .
\end{aligned}
$$

The above results shows that the photon rays are moving in a medium of homogeneous plasma. 


\section{GRAPHICAL ANALYSIS}

This portion is devoted to study the graphical effect of deflection angle $\Theta$ on Horndeski BHs. In addition, we exemplify the physical impact of these graphs to review the effect of plasma term $\frac{\omega_{e}}{\omega_{\infty}}, \tilde{\gamma}$ and impact parameter $b$ on deflection angle.

\section{A. Deflection angle over Impact parameter}

This subsection bestows the analysis of deflection angle $\Theta$ w.r.t impact parameter $b$ for different ranges of parameters.For this, we fixed $\mu=2, \pi=3.14$ and for the sake of simplicity take $\frac{\omega_{e}}{\omega_{\infty}}=\beta$.
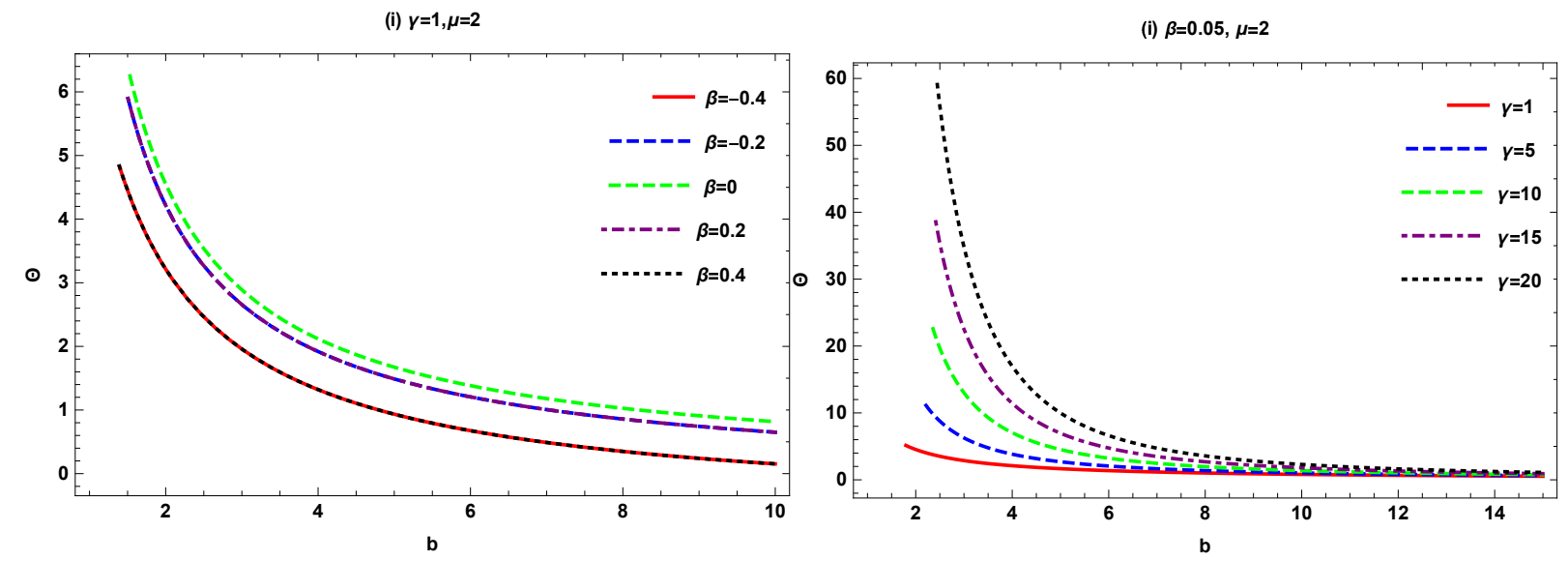

Figure 2: $\Theta$ versus $b$.

- Figure 2 reveals the impact of $\Theta$ over $b$ for different ranges of $\beta$ and $\tilde{\gamma}$ and fixed $\mu$.

1. Figure (i), interpret the graphical effect of deflection angle versus $b$ by varying $\beta$. We investigated that the positive behavior of deflection angle can be analyzed just for $-0.4 \leq \beta \leq 0.4$.

2. Figure (ii), represents that deflection angle exponentially increases by increasing $\tilde{\gamma}$.

\section{CONCLUSION}

In this paper, we have examined the deflection angle of photon by Horndeski $\mathrm{BH}$ in weak field limits. In this connection, we have found the corresponding optical geometry of Horndeski BH. With the aid of this manner, the Gauss-Bonnet theorem has been followed by employing the straight line approximation and enumerate the deflection angle in leading order terms. The acquired angle of deflection is measured by integrating a domain outside the impact parameter, which shows the globally influence of gravitational lensing. Moreover, we have computed the deflection angle of photon by Horndeski $\mathrm{BH}$ in plasma medium. It is to be analyzed that the acquired deflection angle is greater by decreasing the impact parameter similarly the mass term $\mu$ decreases the deflection angle. Hence, it can be visible that the deflection angle increases when the photon rays flow through a medium of homogeneous plasma. Furthermore, we have analyzed the graphical influence of deflection angle on Horndeski BH in non-plasma as well as plasma medium.

\section{Acknowledgments}

This work was supported by Comisión Nacional de Ciencias y Tecnología of Chile through FONDECYT Grant $N^{\circ}$ 3170035 (A. Ö.).

[1] R. H. Sanders and S. S. McGaugh, Ann. Rev. Astron. Astrophys. 40, 263 (2002). 
[2] S. X. Tian and Z. H. Zhu, Phys. Rev. D 100, no. 12, 124059 (2019).

[3] S. Nojiri and S. D. Odintsov, Phys. Lett. B 631, 1 (2005).

[4] E. Berti et al., Class. Quant. Grav. 32, 243001 (2015).

[5] S. Nojiri, S. D. Odintsov and V. K. Oikonomou, Phys. Rept. 692, 1 (2017).

[6] S. Capozziello and M. De Laurentis, Phys. Rept. 509, 167 (2011).

[7] G. W. Horndeski, Int. J. Theor. Phys. 10, 363 (1974).

[8] D. Blas, O. Pujolas and S. Sibiryakov, Phys. Rev. Lett. 104, 181302 (2010).

[9] J. Sakstein and B. Jain, Phys. Rev. Lett. 119, no. 25, 251303 (2017).

[10] R. A. Flores and J. R. Primack, Astrophys. J. 427, L1 (1994)

[11] B. Moore, S. Ghigna, F. Governato, G. Lake, T. R. Quinn, J. Stadel and P. Tozzi, Astrophys. J. 524, L19 (1999)

[12] D. M. Wittman, J. A. Tyson, D. Kirkman, I. Dell'Antonio and G. Bernstein, Nature 405, 143 (2000)

[13] D. Clowe, M. Bradac, A. H. Gonzalez, M. Markevitch, S. W. Randall, C. Jones and D. Zaritsky, Astrophys. J. 648, L109 (2006)

[14] M. Bartelmann and P. Schneider, Phys. Rept. 340, 291 (2001)

[15] N. Kaiser, G. Squires and T. J. Broadhurst, Astrophys. J. 449, 460 (1995)

[16] K. S. Virbhadra and G. F. R. Ellis, Phys. Rev. D 62, 084003 (2000)

[17] V. Bozza, Phys. Rev. D 66, 103001 (2002)

[18] K. S. Virbhadra and G. F. R. Ellis, Phys. Rev. D 65, 103004 (2002).

[19] H. Hoekstra and B. Jain, Ann. Rev. Nucl. Part. Sci. 58, 99 (2008)

[20] C. Y. Peng, C. D. Impey, H. W. Rix, C. S. Kochanek, C. R. Keeton, E. E. Falco, J. Lehar and B. A. McLeod, Astrophys. J. 649, $616(2006)$

[21] K. S. Virbhadra and C. R. Keeton, Phys. Rev. D 77, 124014 (2008)

[22] C. R. Keeton, astro-ph/0102340.

[23] C. R. Keeton and A. O. Petters, Phys. Rev. D 72, 104006 (2005)

[24] A. Bhadra, Phys. Rev. D 67, 103009 (2003)

[25] G. N. Gyulchev and S. S. Yazadjiev, Phys. Rev. D 75, 023006 (2007)

[26] G. W. Gibbons, C. M. Warnick and M. C. Werner, Class. Quant. Grav. 25, 245009 (2008)

[27] G. W. Gibbons and C. M. Warnick, Phys. Rev. D 79, 064031 (2009)

[28] D. C. Latimer, Phys. Rev. D 88, 063517 (2013)

[29] T. Elghozi, N. E. Mavromatos and M. Sakellariadou, Eur. Phys. J. C 77, no. 7, 445 (2017)

[30] W. G. Cao and Y. Xie, Eur. Phys. J. C 78, no. 3, 191 (2018).

[31] Y. K. Lim and Q. h. Wang, Phys. Rev. D 95, no. 2, 024004 (2017)

[32] J. Sultana, JCAP 1304, 048 (2013).

[33] P. Fleury, J. Larena and J. P. Uzan, Phys. Rev. Lett. 119, no. 19, 191101 (2017)

[34] R. Whisker, Phys. Rev. D 71, 064004 (2005)

[35] S. b. Chen and J. 1. Jing, Phys. Rev. D 80, 024036 (2009)

[36] E. F. Eiroa, G. E. Romero and D. F. Torres, Phys. Rev. D 66, 024010 (2002)

[37] C. Y. Wang, Y. F. Shen and Y. Xie, JCAP 1904, 022 (2019)

[38] S. Mao and B. Paczynski, Astrophys. J. 374, L37 (1991).

[39] M. Sharif and S. Iftikhar, Astrophys. Space Sci. 357, no. 1, 85 (2015).

[40] O. Kasikci and C. Deliduman, Phys. Rev. D 100, no. 2, 024019 (2019)

[41] R. Zhang and J. Jing, Eur. Phys. J. C 78, no. 10, 796 (2018).

[42] H. Arakida and M. Kasai, Phys. Rev. D 85, 023006 (2012)

[43] G. S. Bisnovatyi-Kogan and O. Y. Tsupko, Universe 3, no. 3, 57 (2017)

[44] C. Q. Liu, C. K. Ding and J. L. Jing, Chin. Phys. Lett. 34, no. 9, 090401 (2017)

[45] G. S. Bisnovatyi-Kogan and O. Y. Tsupko, Plasma Phys. Rep. 41, 562 (2015)

[46] B. Ahmedov, B. Turimov, Z. Stuchlík and A. Tursunov, Int. J. Mod. Phys. Conf. Ser. 49, 1960018 (2019).

[47] B. Turimov, B. Ahmedov, A. Abdujabbarov and C. Bambi, arXiv:1802.03293 [gr-qc].

[48] A. Abdujabbarov, B. Ahmedov, N. Dadhich and F. Atamurotov, Phys. Rev. D 96, no. 8, 084017 (2017).

[49] J. Schee, Z. Stuchlík, B. Ahmedov, A. Abdujabbarov and B. Toshmatov, Int. J. Mod. Phys. D 26, no. 5, 1741011 (2017).

[50] H. Ghaffarnejad, M. Amirmojahedi and H. Niad, Adv. High Energy Phys. 2018, 3067272 (2018)

[51] A. B. Aazami, C. R. Keeton and A. O. Petters, J. Math. Phys. 52, 102501 (2011)

[52] C. R. Keeton and A. O. Petters, Phys. Rev. D 73, 044024 (2006)

[53] G. W. Gibbons and M. C. Werner, Class. Quant. Grav. 25, 235009 (2008).

[54] M. C. Werner, Gen. Rel. Grav. 44, 3047 (2012).

[55] A. Ishihara, Y. Suzuki, T. Ono, T. Kitamura and H. Asada, Phys. Rev. D 94, no. 8, 084015 (2016).

[56] I. Sakalli and A. Ovgun, EPL 118, no. 6, 60006 (2017).

[57] K. Jusufi, M. C. Werner, A. Banerjee and A. Övgün, Phys. Rev. D 95, no. 10, 104012 (2017).

[58] T. Ono, A. Ishihara and H. Asada, Phys. Rev. D 96, no. 10, 104037 (2017).

[59] K. Jusufi, I. Sakalli and A. Övgün, Phys. Rev. D 96, no. 2, 024040 (2017).

[60] A. Ishihara, Y. Suzuki, T. Ono and H. Asada, Phys. Rev. D 95, no. 4, 044017 (2017).

[61] K. Jusufi, A. Övgün and A. Banerjee, Phys. Rev. D 96, no. 8, 084036 (2017).

[62] H. Arakida, Gen. Rel. Grav. 50, no. 5, 48 (2018).

[63] K. Jusufi and A. Övgün, Phys. Rev. D 97, no. 2, 024042 (2018).

[64] A. Övgün, I. Sakalli and J. Saavedra, JCAP 1810, no. 10, 041 (2018) 
[65] A. Övgün, I. Sakalli and J. Saavedra, Annals Phys. 411, 167978 (2019).

[66] A. Övgün, G. Gyulchev and K. Jusufi, Annals Phys. 406, 152 (2019).

[67] K. Jusufi, A. Övgün, J. Saavedra, P. A. Gonzalez and Y. Vasquez, Phys. Rev. D 97, 124024 (2018).

[68] A. Övgün, K. Jusufi and I. Sakalli, Annals Phys. 399, 193 (2018).

[69] K. Jusufi and A. Övgün, Phys. Rev. D 97, no. 6, 064030 (2018).

[70] A. Övgün, Phys. Rev. D 98, no. 4, 044033 (2018)

[71] T. Ono, A. Ishihara and H. Asada, Phys. Rev. D 98, no. 4, 044047 (2018)

[72] T. Ono, A. Ishihara and H. Asada, Phys. Rev. D 99, no. 12, 124030 (2019).

[73] G. Crisnejo and E. Gallo, Phys. Rev. D 97, 124016 (2018).

[74] G. Crisnejo, E. Gallo and A. Rogers, Phys. Rev. D 99, no. 12, 124001 (2019).

[75] Z. Li and A. Övgün, doi:10.20944/preprints201911.0195.v1

[76] Z. Li and T. Zhou, arXiv:1908.05592 [gr-qc].

[77] Z. Li and J. Jia, arXiv:1912.05194 [gr-qc].

[78] Z. Li, G. He and T. Zhou, arXiv:1908.01647 [gr-qc].

[79] K. Jusufi, A. Övgün, A. Banerjee and I. Sakalli, Eur. Phys. J. Plus 134, no. 9, 428 (2019).

[80] A. Övgün, Universe 5, no. 5, 115 (2019).

[81] W. Javed, R. Babar, A. Ovgun, Phys. Rev. D 99, no. 8, 084012 (2019).

[82] W. Javed, A. Hazma and A. Övgün, Preprints 2019, 2019110142 (doi:10.20944/preprints201911.0142.v1)

[83] W. Javed, J. Abbas, and A. Övgün, Eur. Phys. J. C 79, no. 8, 694 (2019).

[84] A. Övgün, Phys. Rev. D 99, no. 10, 104075 (2019).

[85] W. Javed, j. Abbas and A. Övgün, Phys. Rev. D 100, no. 4, 044052 (2019).

[86] K. Jusufi and A. Övgün, Int. J. Geom. Meth. Mod. Phys. 16, no. 08, 1950116 (2019).

[87] G. Crisnejo, E. Gallo, and J. R. Villanueva, Phys. Rev. D 100, no. 4, 044006 (2019).

[88] W. Javed, R. Babar, and A. Övgün, Phys. Rev. D 100, no. 10, 104032 (2019)

[89] K. de Leon and I. Vega, Phys. Rev. D 99, no. 12, 124007 (2019)

[90] Y. Kumaran and A. Ovgün, arXiv:1905.11710 [gr-qc].

[91] W. Javed, J. Abbas, Y. Kumaran and A. Övgün, Preprints 2019, 2019110210 (doi: 10.20944/preprints201911.0210.v1).

[92] A. Övgün, I. Sakalli and J. Saavedra, arXiv:1908.04261 [gr-qc].

[93] Z. Li and A. Övgün, Preprints 2019, 2019110195 (doi: 10.20944/preprints201911.0195.v1)

[94] E. Babichev, C. Charmousis and A. Lehébel, JCAP 1704, no. 04, 027 (2017).

[95] J. Badia and E. F. Eiroa, Eur. Phys. J. C 77, no. 11, 779 (2017).

[96] G.W. Gibbons and M. C.Werner, Class. Quant. Grav. 25, (2008)235009. 